\title{
Schwinger's Oscillator Method, Supersymmetric Quantum Mechanics and Massless Particles
}

\author{
F. M. Mejía ${ }^{1}$ and V. Pleitez ${ }^{2}$ \\ ${ }^{1}$ Escuela de Física, Facultad de Ciencias Naturales y Matemática \\ Universidad de El Salvador, El Salvador \\ ${ }^{2}$ Instituto de Física Teórica, Universidade Estadual Paulista \\ Rua Pamplona 145, 01405-900, São Paulo, SP, Brazil
}

Recebido em 05 de novembro 2001. Aceito em 11 de dezembro, 2001.

\begin{abstract}
We consider Schwinger's method of angular momentum addition using the $S U(2)$ algebra with both a fermionic and a bosonic oscillator. We show that the total spin states obtained are: one boson singlet state and an arbitrary number of spin- $1 / 2$ states, the later ones are energy degenerate. It means that we have in this case supersymmetric quantum mechanics and also the addition of angular momentum for massless particles. We review too the cases of two bosonic and two fermionic oscillators.
\end{abstract}

\section{Introduction}

The usual method for defining the angular momentum in quantum mechanics is by means of the commutation relations satisfied by its components $J_{i}, i=x, y, z$; and by solving the eigenvalue problem for $\vec{J}^{2}$ and $J_{z}$ assuming that the components $J_{i}$ are observables. From this, the allowed values for the eigenvalues of $\vec{J}^{2}$ and $J_{z}$, denoted $j$ and $m$, respectively, are obtained. They run over the values: $j=0,1 / 2,1,3 / 2, \ldots$ and $-j \leq m \leq$ $j$ [1]. In this case the angular momentum operators $J_{i}$ are the infinitesimal generators for the $S O(3) \sim S U(2)$ algebra. The relation between spin and $S U(2)$ symmetry is maintained in relativistic field theory since the little group for massive particles is just the rotation group $[2,3]$. For massive spin- $j$ particles we can always go to the rest frame, thus their spin degrees of freedom transform according to a $(2 j+1)$-dimensional representation of $S U(2)$, that is, we have $2 j+1$ polarization states.

In the case of massless particles it is not possible to go to the rest frame, so the spin is no longer described by $S U(2)$. In this case the little group is the Euclidean $I S O(2)$, denoted also by $E(2)$. This group consists of rotations by an angle $\phi$ around the $z$-direction (assuming this as the direction of the motion) and translations in the Euclidean plane perpendicular to the $z$ axis. Its irreducible representations must either represent the translation by zero, or be infinite-dimensional. If $\chi$ is an eigenvector of the translation generators, $e^{i \phi J_{z}} \chi$ will be also an eigenvector rotated by an angle $\phi$. There is no room in physics besides the momentum for another continuous quantum number, so physical massless particles correspond only to the first kind of representations (i.e., the trivial ones) [3]. This leaves only $J_{z}$ as symmetry operator, so the physical representations of $E(2)$ are one-dimensional and labeled by the helicity $\lambda$. $J_{z}|\lambda\rangle=\lambda|\lambda\rangle$. This is why the polarization states of a massless particle with spin $j$ are only $\pm j$. We can see this by considering the second Casimir invariant $W^{\mu} W_{\mu}=-M^{2} j(j+1)$ (the other is $p^{2}=M^{2}>0$ ) where $W^{\mu}$ is the Pauli-Lubanski pseudovector defined as

$$
W_{\mu}=-\frac{1}{2} \varepsilon_{\mu \nu \rho \sigma} J^{\nu \rho} P^{\sigma},
$$

with $J^{\nu \rho}$ and $P^{\sigma}$ denoting the generators of the Poincaré group; $\varepsilon_{\mu \nu \rho \sigma}$ is the totally antisymmetric symbol in four dimensions. Since $M^{2}=0$ we have for a state of the four momentum $k$,

$$
W \cdot W|k\rangle=0, \quad P \cdot P|k\rangle=0
$$

and, since $W^{\mu} P_{\mu}=0$,

$$
W \cdot P|k\rangle=0 .
$$

So, $W^{\mu}$ and $P^{\mu}$ are orthogonal and both lightlike. This means that they must be proportional

$$
\left(W^{\mu}-\lambda P^{\mu}\right)|k\rangle=0,
$$

and we have the result that the state of a massless particle is characterized by one number $\lambda$, which is the ratio of $W^{\mu}$ and $P^{\mu}$ and so it has the dimension of angular momentum. It is called, as we said before, helicity. If parity is included the helicity takes on two values, $\lambda$ and $-\lambda$. The fact that $\lambda$ can be integral or half-integral is 
due to the fact that $e^{i 4 \pi \lambda}$ must be unity, and hence $\lambda$ must be integer or half-integer [3].

Several years ago Schwinger worked out a connection between the algebra of angular-momentum and two uncoupled bosonic oscillators [4]. The Schwinger's scheme permits to calculate the eigenvalues $j$ and $m$, in terms of the operator number of the uncoupled oscillators. The results agree with the general method for massive particles discussed above. The case of massless particles, however, does not arise within Schwinger's scheme. So, the method must be generalized. In this work we take a first step to a more complete generalization by considering one or more fermionic oscillators.

The outline of this work is the following. Schwinger's method which consists of two bosonic oscillators $(B B)$ is reviewed in Sec.II. Next, we generalize the scheme by considering i) two fermionic oscillators $(F F)$ in Sec. III and, ii) one bosonic and one fermionic oscillator $(F B)$ in Sec. IV. In the last cases only spin zero and spin $1 / 2$ are generated. In the last section we show that in the BF case the spin $1 / 2$ particles are infinitely degenerated and supersymmetric quantum mechanics naturally arises.

\section{Two bosonic oscillators (BB)}

In this section we will briefly review the Schwinger scheme $[4,5]$ by considering two simple bosonic oscillators with annihilation and creation operators $a_{i}$ and $a_{i}^{\dagger}, i=1,2$, respectively. The number operators are (throughout this work we will use $\hbar=1$ ) $N_{i} \equiv a_{i}^{\dagger} a_{i}$ and assuming the commutation relations $\left[a_{i}, a_{j}^{\dagger}\right]=\delta_{i j}$, it follows that

$\left[N_{i}, a_{j}\right]=-a_{i} \delta_{i j}, \quad\left[N_{i}, a_{j}^{\dagger}\right]=a_{i}^{\dagger} \delta_{i j}, \quad$ (no summation).

We also assume that another pair of operators of the same oscillator or of different oscillators commute. It means that the two oscillators are uncoupled. Because $N_{1}$ and $N_{2}$ commute, we can build up simultaneous eigenstates of $N_{1}$ and $N_{2}$ with eigenvalues $n_{1}$ and $n_{2}$, respectively.

Next, we define

$$
J_{+} \equiv a_{1}^{\dagger} a_{2}, \quad J_{-} \equiv a_{2}^{\dagger} a_{1},
$$

and

$$
J_{z} \equiv \frac{1}{2}\left(a_{1}^{\dagger} a_{1}-a_{1}^{\dagger} a_{2}\right)=\frac{1}{2}\left(N_{1}-N_{2}\right)
$$

These operators satisfy the $S U(2)$ commutation relations

$$
\left[J_{z}, J_{ \pm}\right]= \pm J_{ \pm}, \quad\left[J_{+}, J_{-}\right]=2 J_{z}
$$

Defining the total number operator $N$ (with eigenvalues $n_{1}+n_{2}$ )

$$
N \equiv N_{1}+N_{2}=a_{1}^{\dagger} a_{1}+a_{2}^{\dagger} a_{2},
$$

it follows that the quadratic Casimir operator

$$
\vec{J}^{2}=J_{z}^{2}+\frac{1}{2}\left(J_{+} J_{-}+J_{-} J_{+}\right)
$$

can be written as

$$
\vec{J}^{2}=\frac{N}{2}\left(\frac{N}{2}+1\right) .
$$

If we associate spin up $(m=1 / 2)$ with one quantum unit of the $N_{1}$ oscillator and spin down $(m=-1 / 2)$ with one quantum unit of the $N_{2}$ oscillator, it is possible to imagine a spin $1 / 2$ "particle" with spin up (down) with each quantum unit of the $N_{1}\left(N_{2}\right)$ oscillator. The eigenvalues $n_{1}$ and $n_{2}$ are just the number of spin up and spin down "particles", respectively. We will see that the association of half-integral spin with bosonic oscillators is necessary, if we want to construct a general $|j, m\rangle$ state with $j=0,1 / 2,1,3 / 2,2, \ldots$ and $-j \leq m \leq j$.

Turning back to the $J_{ \pm}$operators defined in Eqs. (II), we see that $J_{+}$destroys one unit of spin down with the $z$-component of angular $-1 / 2$ and creates one unit of spin up with the $z$-component of angular momentum $+1 / 2$. So, the $z$-component of angular momentum is therefore increased by 1 . Likewise $J_{-}$destroys one unit of spin up and creates one unit of spin down, the $z$-component of angular momentum is therefore decreased by 1 . As for the $J_{z}$ operator, it simply counts $1 / 2(\hbar=1)$ times the difference between $n_{1}$ and $n_{2}$, just gives the $z$-component of the total angular momentum. Hence, the action of the $J_{ \pm}$and $J_{z}$ operators on the eigenstates of the $\left|n_{1}, n_{2}\right\rangle$ is given by

$$
\begin{gathered}
J_{+}\left|n_{1}, n_{2}\right\rangle=a_{1}^{\dagger} a_{2}\left|n_{1}, n_{2}\right\rangle=\left[n_{2}\left(n_{1}+1\right)\right]^{1 / 2}\left|n_{1}+1, n_{2}-1\right\rangle, \\
J_{-}\left|n_{1}, n_{2}\right\rangle=a_{2}^{\dagger} a_{1}\left|n_{1}, n_{2}\right\rangle=\left[n_{1}\left(n_{2}+1\right)\right]^{1 / 2}\left|n_{1}-1, n_{2}+1\right\rangle, \\
J_{z}\left|n_{1}, n_{2}\right\rangle=\frac{1}{2}\left(N_{1}-N_{2}\right)\left|n_{1}, n_{2}\right\rangle=\frac{1}{2}\left(n_{1}-n_{2}\right)\left|n_{1}, n_{2}\right\rangle .
\end{gathered}
$$

Notice that, the sum $n_{1}+n_{2}$ which gives the total number of spin $1 / 2$ particles remains unchanged. If we substitute

$$
n_{1} \rightarrow j+m, \quad n_{2} \rightarrow j-m,
$$


Eqs. () reduce to the usual factors

$$
\begin{gathered}
J_{+}\left|n_{1}, n_{2}\right\rangle=[(j-m)(j+m+1)]^{1 / 2}|j+m+1, j-m-1\rangle, \\
J_{-}\left|n_{1}, n_{2}\right\rangle=[(j+m)(j-m+1)]^{1 / 2}|j+m-1, j-m+1\rangle, \\
J_{z}|j+m, j-m\rangle=m|j+m, j-m\rangle,
\end{gathered}
$$

and the eigenvalues of the quadratic Casimir operator $\vec{J}^{2}$ defined in Eq. (10) become

$$
\begin{aligned}
\vec{J}^{2}|j+m, j-m\rangle & =\frac{n_{1}+n_{2}}{2}\left[\frac{n_{1}+n_{2}}{2}+1\right]|j+m, j-m\rangle \\
& \equiv j(j+1)|j+m, j-m\rangle .
\end{aligned}
$$

The connection between the oscillator matrix elements and angular momentum matrix elements can be seen by defining

$$
j \equiv \frac{n_{1}+n_{2}}{2}, \quad m \equiv \frac{n_{1}-n_{2}}{2},
$$

in place of $n_{1}$ and $n_{2}$ to characterize simultaneous eigenkets of $\vec{J}^{2}$ and $J_{z}$. Hence, the most general $N_{1}, N_{2}$ eigenket is

$$
|j, m\rangle=\frac{\left(a_{1}^{\dagger}\right)^{j+m}\left(a_{2}^{\dagger}\right)^{j-m}}{[(j+m) !(j-m) !]^{1 / 2}}|0,0\rangle .
$$

If $j=m$ we have the largest eigenvalue for $J_{z}$

$$
|j, j\rangle=\frac{\left(a_{1}^{\dagger}\right)^{2 j}}{[(2 j) !]^{1 / 2}}|0,0\rangle
$$

so we can imagine this state to be build up of $2 j$ spin $1 / 2$ particles with their spin all pointing in the positive $z$-direction. Hence, as we said before, in this scheme an object of high $j$ can be visualized as being made up of primitive spin 1/2 "particles", $j+m$ of them with spin up and the remaining $j-m$ of them with spin down. This of course, does not mean that an object of angular momentum $j$ is a composite system of spin $1 / 2$ particles. It means only that, as far as the transformation properties are concerned, we can visualized any object of arbitrary angular momentum $j$ as a composite system of $2 j$ spin $1 / 2$ particles formed in the manner indicated by Eq. (20). This is the well known Schwinger's oscillator method [5].

\section{Two fermionic oscillators (FF)}

Let us consider two fermionic oscillators with annihilation and creation operators denoted by $F_{i}$ and $F_{i}^{\dagger}$, $i=1,2$. Then

$$
\left\{F_{i}, F_{j}^{\dagger}\right\}=\delta_{i j},
$$

and any other pair of operators anticommuting. The number operators are defined as usual $N_{i}=F_{i}^{\dagger} F_{i}, i=1$ or 2 , and they satisfy

$\left[N_{i}, F_{j}\right]=-F_{i} \delta_{i j}, \quad\left[N_{i}, F_{j}^{\dagger}\right]=F_{i}^{\dagger} \delta_{i j}$, (no summation).

However, from Eq. (22) it follows that

$$
N_{i}\left(N_{i}-1\right)=0, \quad i=1 \text { or } 2 ;
$$

so, the only eigenvalues of $N_{i}$, denoted by $n_{i}$, are 0 or 1 . The total number operator $N=\sum_{i} N_{i}$, has eigenvalues 0,1 or 2 .

As in the case of two bosonic oscillators, we can construct simultaneous eigenkets of $N_{1}$ and $N_{2}$. Eqs. (21) are valid but with the substitution $a_{i} \rightarrow F_{i}, a_{i}^{\dagger} \rightarrow F_{i}^{\dagger}$ and with the constraint upon $n_{1}, n_{2}$ given above. Thus as in Eqs. (6) we can define

$$
\begin{gathered}
J_{+} \equiv F_{1}^{\dagger} F_{2}, \quad J_{-} \equiv F_{2}^{\dagger} F_{1}, \\
J_{z} \equiv \frac{1}{2}\left(F_{1}^{\dagger} F_{1}-F_{2}^{\dagger} F_{2}\right)=\frac{1}{2}\left(N_{1}-N_{2}\right),
\end{gathered}
$$

which satisfy, as can be easily verified, the $S U(2)$ commutation relations in Eq. (7). From the point of view of the $S U(2)$ algebra both cases, two bosonic oscillators and two fermionic oscillators are equivalent. Notwithstanding $\vec{J}^{2}$, defined in Eq. (9), when written in terms of the number operators, instead of Eq. (10) is given by

$$
\vec{J}^{2}=\frac{N}{2}\left(\frac{N}{2}+1\right)-2 N_{1} N_{2} .
$$

Since the eigenvalues of $N_{1}$ and $N_{2}$ can assume only the values 0 or 1 , we see from $\mathrm{Eq}$. (27) that the respective eigenvalues for $\vec{J}^{2}$ are 0 and $3 / 4$. If we interpret these values in the form $j(j+1), j \geq 0$, we see that only $j=0$ and $j=1 / 2$ are allowed. The eigenvalues of $J_{z}$ defined in Eq. (26) are 0,1/2,-1/2 [6]: 


$$
\begin{gathered}
J_{z}|0,0\rangle=0, \quad J_{z} F_{1}^{\dagger} F_{2}^{\dagger}|0,0\rangle=0, \quad J_{z}|1,1\rangle=0, \quad J^{2}|1,1,\rangle=0, \\
J_{z} F_{1}^{\dagger}|0,0\rangle=\frac{1}{2} F_{1}^{\dagger}|0,0\rangle, \quad J_{z} F_{2}^{\dagger}|0,0\rangle=-\frac{1}{2} F_{2}^{\dagger}|0,0\rangle .
\end{gathered}
$$

Hence, it seems that with two fermionic oscillators we can build up only one spin-1/2 $\left.\left(F_{1}^{\dagger}|0,0\rangle,\left|F^{\dagger}\right| 0,0\right\rangle\right)$ and two spin- 0 states $(|0,0\rangle,|1,1\rangle)$. In others words, although the system also satisfies the usual angularmomentum commutation relations in Eq. (7), only these two values for the total angular-momentum are allowed. We call this situation a constrained realization of the $S U(2)$ algebra.

If we associate spin up $(m=1 / 2)$ with one quantum unit of the $F_{1}$ oscillator and spin down $(m=-1 / 2)$ with one quantum unit of the $F_{2}$ oscillator, it is possible to imagine one spin 1/2 "particle" with spin up (down) with each quantum unit of the $F_{1}\left(F_{2}\right)$ oscillator. As in Sec. II the spins are along the $z$-direction and the eigenvalues $n_{1}$ and $n_{2}$ are just the numbers of spins up and spins down, respectively. However, in the present case if $n_{1}=n_{2}=0$ the total spin is also zero; if $n_{1}=n_{2}=1$ both spins are in opposite direction and the total spin vanishes again. On the other hand, if $n_{1}=1, n_{2}=0$ the total spin is $1 / 2$ and the projection on the $z$-axis is $1 / 2$; if $n_{1}=0, n_{2}=1$ the total spin is again $1 / 2$ but its projection in that axis is $-1 / 2$. Notice, however, that this case does not correspond neither to the massive nor to the massless particle cases. It can be applied to both kinds of particles.

\section{One fermionic and one bosonic oscillators (FB)}

We have seen that both, the usual angular momentum addition and the Schwinger's scheme are valid for the case of massive particles [5]. The case of massless particles, however, does not arise neither within the usual approach nor in the Schwinger's scheme. So, the method must be generalized. In this work we take a first step to get a more complete generalization by considering one fermionic oscillator.

The interesting feature of Schwinger's scheme is that it allows us to obtain what are the values of the weights or roots that are realized in the $S U(2)$ algebra. For instance, in the original work of Schwinger, all representations of the $S U(2)$ algebra arise and are exactly equivalent, as we said before, to the theory of the angular momentum addition. This is however a consequence of the bosonic nature of the oscillators. Notwithstanding, when both oscillators are fermionic, although the algebraic relations are still valid the method does not coincide with the usual addition of angular momentum in the sense that only restricted values for the eigenvalues of the angular momentum operator are allowed: only two spinless states and one spin- $1 / 2$ state are obtained and it is impossible to recover the full set of the unitary representation of $S U(2)$. This is a consequence of the fermionic character of the operators with which we implement the realization of the $S U(2)$ algebra [6]. On the other hand, when one of the oscillators is bosonic and the other one is fermionic, a usual $S U(2)$ algebra is still realized but also in a restricted sense. This is the case that we will consider here.

Let us consider the case of two oscillators, one of them a bosonic oscillator $\left(a, a^{\dagger}\right)$ and the other one a fermionic oscillator $\left(F, F^{\dagger}\right)$. It means that

$$
\left[a, a^{\dagger}\right]=1, \quad\left\{F, F^{\dagger}\right\}=1,
$$

and any pair of operators commutes if both of them are bosonic operators or, if one of them is a bosonic operator and the other is a fermionic operator; they anticommute if both of them are fermionic operators.

As before, we will use the following notation: The number operators are denoted by $N_{B}=a^{\dagger} a, N_{F}=$ $F^{\dagger} F$ and $N=N_{B}+N_{F}$, with eigenvalues $n_{B}, n_{F}$ and $n=n_{B}+n_{F}$, respectively. Let us define

$$
\begin{gathered}
J_{+} \equiv a^{\dagger} F\left(N_{B}+1\right)^{-1 / 2}, \quad J_{-} \equiv\left(N_{B}+1\right)^{-1 / 2} F^{\dagger} a, \\
J_{z} \equiv \frac{1}{2}\left[a^{\dagger}\left(N_{B}+1\right)^{-1} a\left(1-N_{F}\right)-N_{F}\right] .
\end{gathered}
$$


If the simultaneous eigenkets of $N_{B}$ and $N_{F}$ are denoted by $\left|n_{B}, n_{F}\right\rangle$ when necessary we will use the closure relation $\sum\left|n_{B}, n_{F}\right\rangle\left\langle n_{B}, n_{F}\right|=\mathbf{1}$ in order to get a result that it is not state dependent. For instance

$$
\left(N_{B}+1\right)^{-1} \sum_{n_{B}, n_{F}}\left|n_{B}, n_{F}\right\rangle\left\langle n_{B}, n_{F}\left|=\sum_{n_{B}, n_{F}}\left(n_{B}+1\right)^{-1}\right| n_{B}, n_{F}\right\rangle\left\langle n_{B}, n_{F}\right|,
$$

and similarly for $\left(N_{B}+1\right)^{-1 / 2}$. Then, it is possible to verify that the operators defined in Eq. (32) satisfy the commutation relations of the $S U(2)$ algebra given in Eq. (7) and that

$$
J_{+}\left|n_{B}, n_{F}\right\rangle=a^{\dagger}\left(N_{B}+1\right)^{-1 / 2} F\left|n_{B}, n_{F}\right\rangle=\sqrt{n_{F}}\left|n_{B}+1, n_{F}-1\right\rangle
$$

that is, $J_{+}\left|n_{B}, n_{F}\right\rangle=\left|n_{B}+1,0\right\rangle$ if $n_{F}=1$ and $J_{+}\left|n_{B}, n_{F}\right\rangle=0$ if $n_{F}=0$. Similarly,

$$
J_{-}\left|n_{B}, n_{F}\right\rangle=F^{\dagger}\left(N_{B}+1\right)^{-1 / 2} a\left|n_{B}, n_{F}\right\rangle=\sqrt{1-n_{F}}\left|n_{B}-1, n_{F}+1\right\rangle,
$$

hence $J_{-}\left|n_{B}, n_{F}\right\rangle=0$ when $n_{F}=1$ or $n_{B}=0$, and $J_{-}\left|n_{B}, n_{F}\right\rangle=\left|n_{B}-1,1\right\rangle$ when $n_{F}=0$.

Next, we obtain the quadratic Casimir operator

$$
\vec{J}^{2}=\frac{1}{2}\left(\frac{1}{2}+1\right)\left[a^{\dagger}\left(N_{B}+1\right)^{-1} a\left(1-N_{F}\right)+N_{F}\right],
$$

and the hamiltonian of the system can be written as

$$
H=\left\{J_{+}, J_{-}\right\}=a^{\dagger}\left(N_{B}+1\right)^{-1} a\left(1-N_{F}\right)+N_{F},
$$

and it satisfies $\left[H, J_{ \pm}\right]=0$.

For the state $\left|n_{B}, n_{F}\right\rangle=|0,0\rangle$ we have

$$
J_{z}|0,0\rangle=0, J^{2}|0,0\rangle=0,
$$

while for the states $\left|n_{B}, n_{F}\right\rangle \neq|0,0\rangle$ it follows

$$
J_{z}\left|n_{B}, n_{F}\right\rangle=\left(\frac{1}{2}-n_{F}\right)\left|n_{B}, n_{F}\right\rangle,
$$

and

$$
J^{2}\left|n_{B}, n_{F}\right\rangle=\frac{1}{2}\left(\frac{1}{2}+1\right)\left|n_{B}, n_{F}\right\rangle .
$$

We see from Eqs. (38) and (40) that as in FF case, only spin 0 and $1 / 2$ are generated. However an interesting difference appears when one of the oscillators is bosonic, as we will see in the next section.

\section{Conclusions}

In the usual supersymmetric quantum mechanics, the equality $\omega_{B}=\omega_{F}$ is imposed by hand [7]. In the Schwinger's scheme it is implicitly assumed that both oscillators have the same frequency, $\omega$. In the case of both one fermionic and one bosonic oscillator this implies that $\omega_{B}=\omega_{F}$. It means that we have a symmetry of the combined bosonic and fermionic oscillators, that is, we have a supersymmetry. In fact, using Eq. (37), we have

$$
H|0,0\rangle=0, \quad H\left|n_{B}, n_{F}\right\rangle=\left|n_{B}, n_{F}\right\rangle .
$$

So, we can identify $Q=J_{+}$and $\bar{Q}=J_{-}$as the supersymmetry generators. Note that, in fact, from Eqs.(34) and (35) we have

$$
J_{+}\left|n_{B}, 1\right\rangle=\left|n_{B}+1,0\right\rangle, \quad J_{-}\left|n_{B}+1,0\right\rangle=\left|n_{B}, 1\right\rangle .
$$

Then, the bosonic states $\left|n_{B}+1,0\right\rangle$ have the same energy than their fermionic partners $\left|n_{B}, 1\right\rangle$. Only the vacuum state is not degenerate as it can be seen from Table I.

Table I. States obtained by the Schwinger scheme and the respective energies.

\begin{tabular}{||c|c||c|c|c||}
\hline$n_{B}$ & $n_{F}$ & $j$ & $m$ & $E$ \\
\hline 0 & 0 & 0 & 0 & 0 \\
& 1 & $1 / 2$ & $-1 / 2$ & 1 \\
1 & 0 & $1 / 2$ & $1 / 2$ & 1 \\
& 1 & $1 / 2$ & $-1 / 2$ & 1 \\
2 & 0 & $1 / 2$ & $1 / 2$ & 1 \\
& 1 & $1 / 2$ & $-1 / 2$ & 1 \\
$\cdots$ & $\cdots$ & $\cdots$ & $\cdots$ & $\cdots$ \\
\hline
\end{tabular}

In fact, since in this case we have $N=1$ supersymmetric quantum mechanics, we can introduce a Grassmann parameter $\theta\left(\theta^{2}=0\right)$, and if we define

$$
J_{1}=\theta\left(J_{+}+J_{-}\right), \quad J_{2}=i \theta\left(J_{+}-J_{-}\right),
$$

we can verify that

$$
\left[J_{z}, J_{1}\right]=i J_{2}, \quad\left[J_{z}, J_{2}\right]=-i J_{1}
$$

and

$$
\left[J_{1}, J_{2}\right]=-4 i \theta^{2} J_{z}=0 .
$$

This commutation relation defines the Euclidean group $E(2)$ and, as we mentioned in Sec. I, it is well known 
that in the relativistic theory this is the little group related to massless particles [3] and for this reason the polarization states of massless particles with spin $j$ are only $\pm j$. Thus we can interpret our result as follows: since we are in a non-relativistic domain, i.e. just one $\mathrm{SU}(2)$, only spinless and spin one-half particles are allowed to be massless since in this case both $2 j+1$ or $\pm j$ degeneration coincide. From the point of view of the angular momentum addition, a supersymmetric transformation

$$
\left|n_{B}+1,0\right\rangle \Longleftrightarrow\left|n_{B}, 1\right\rangle,
$$

is equivalent to a parity transformation which makes $j \rightarrow-j$ and supersymmetric quantum mechanics is equivalent to a "constrained $\mathrm{SU}(2)$ algebra".

If we consider $S U(2) \otimes S U(2)^{\prime}$, which corresponds to the relativistic case, we can have massless states with $j=0,1 / 2,1$ but not with $j>1$. A way to overcome this problem is to consider a two-component spinor field as in Ref. [6] as we will show elsewhere.

Finally, we would like to pointed out that it may be interesting to considered these extensions of the Schwinger scheme for $S U(n)$ and also in relativistic field theories [8].

\section{Acknowledgements}

This work was supported partially by Fundação de Amparo à Pesquisa do Estado de São Paulo (FAPESP), Conselho Nacional de Ciência e Tecnologia (CNPq) and by Programa de Apoio a Núcleos de Excelência (PRONEX). One of us (VP) would like to thank J. F. Gomez for calling his attention to Refs. [6, 7].

\section{References}

[1] A. Messiah, Quantum Mechanics, (North-Holland, New York, 1966); Chap. XII.

[2] E. Wigner, "On Unitary Representations of the Inhomogeneous Lorentz Group", Ann. Math. 40 149-204 (1932). This paper is reprinted in Symmetry Groups in Nuclear and Particle Physics, edited by F. J. Dyson (Benjamin, New York, 1966).

[3] S. Weinberg, The Quantum Theory of Fields, (Cambridge University Press, 1995); Vol. I.

[4] J. Schwinger, "On Angular Momentum", in Quantum Theory of Angular Momentum, edited by L. C. Biedenharn and H. Van Dam, (Academic Press, New York, 1965); pag. 229.

[5] J. J. Sakurai, Modern Quantum Mechanics, (AddisonWesley, New York, 1990); pag. 217.

[6] A. Lerda and S. Sciuto, "Anyons and Quantum Groups," Nucl. Phys. B401, 613-643 (1993).

[7] For a review of supersymmetric quant um mechanics see F. Cooper, A. Khare and U. Sukhatne, "Supersymmetry and Quantum-Mechanics," Phys. Rep. 251, 267385 (1995). See also E. Drigo Filho, "Supersimetria em Mecânica Quântica," Rev. Bra. Ens. Fis. 19, 152-158 (1997).

[8] J. Schwinger, "Multispinor basis of Fermi-Bose Transformations", Ann. Phys. (N.Y.) 119, 192-237 (1975). 